\title{
A 2:1 Ratio Inductive Voltage Divider With Less Than 0.1 PPM Error to $1 \mathrm{MHz}$
}

\author{
Cletus A. Hoer* and Walter L. Smith* \\ Institute for Basic Standards, National Bureau of Standards, Boulder, Colo. 80302
}

(October 25, 1966)

\begin{abstract}
A simple ratio transformer having a $2: 1$ ratio of input voltage to output voltage can be made with a ratio error less than $0.1 \mathrm{ppm}$ from $1 \mathrm{kHz}$ to above $1 \mathrm{MHz}$. Applications and sources of error are discussed. Experimental results leading to an optimum transformer design are given. A bridge to measure the ratio error to $0.025 \mathrm{ppm}$ from $1 \mathrm{kHz}$ to $1 \mathrm{MHz}$ is also described.
\end{abstract}

Key Words: Attenuator, bridge, high frequency, inductive voltage divider, ratio transformer.

\section{Introduction}

Techniques for winding inductive voltage dividers with adjustable ratios have been improved during the last few years to where dividers having errors less than $0.1 \mathrm{ppm}$ at frequencies around $1 \mathrm{kHz}$ are not too difficult to make. Unfortunately, as the frequency increases, the ratio error increases rapidly so that this accuracy cannot be maintained above about $10 \mathrm{kHz}$. At frequencies above $10 \mathrm{kHz}$ the most accurate ratio device is an inductive voltage divider which has a fixed voltage ratio of $2: 1$; that is, one dividing an input voltage into two equal parts such as is shown in figure 1 . Such dividers have recently been made with errors less than $0.1 \mathrm{ppm}$ from $1 \mathrm{kHz}$ to above $1 \mathrm{MHz}$. This paper summarizes the research that was done to develop this accurate high frequency divider.

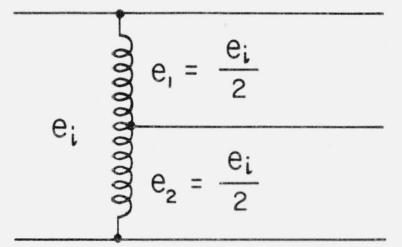

FIGURE 1. A 2:1 ratio inductive voltage divider.

\section{Applications}

A few of the many possible applications for this type of divider are shown in figure 2. Figure 2a is a bridge for comparing two impedances, $z_{1}$ and $z_{2}$.

*Radio Standards Engineering Division, NBS Laboratories, Boulder, Colo. 80302.

Figure $2 \mathrm{~b}$ shows $n$ basic 2:1 ratio dividers connected to give a voltage ratio

$$
\frac{V_{\text {out }}}{V_{\text {in }}}=\frac{1}{2^{n}}
$$

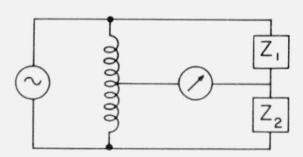

(a)

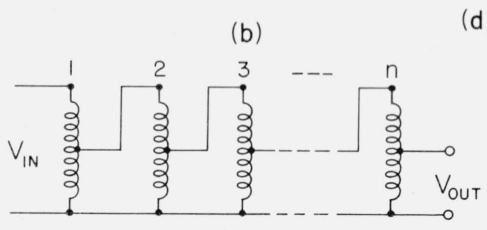

$$
\frac{V_{\text {OUT }}}{V_{\text {IN }}}=\frac{1}{2^{n}} \quad \frac{V_{1}}{V_{2}}=1,2 \text {, or } 5
$$$$
Z_{1}=Z_{2}
$$

d)

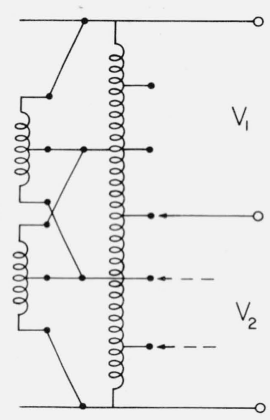

(c)

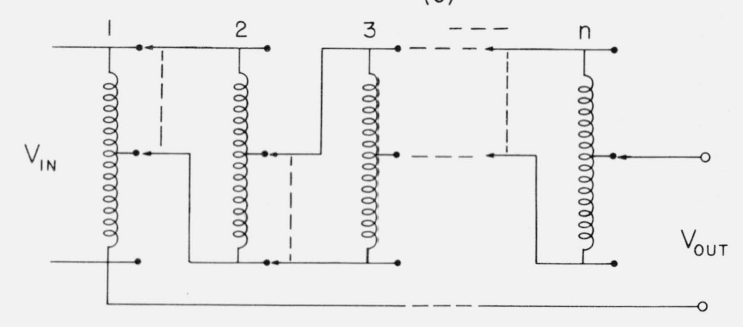

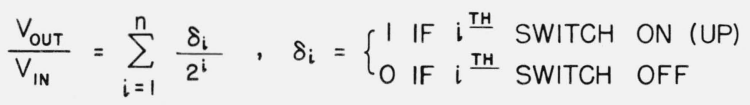

FIGURE 2. Some possible applications for 2:1 ratio dividers. 

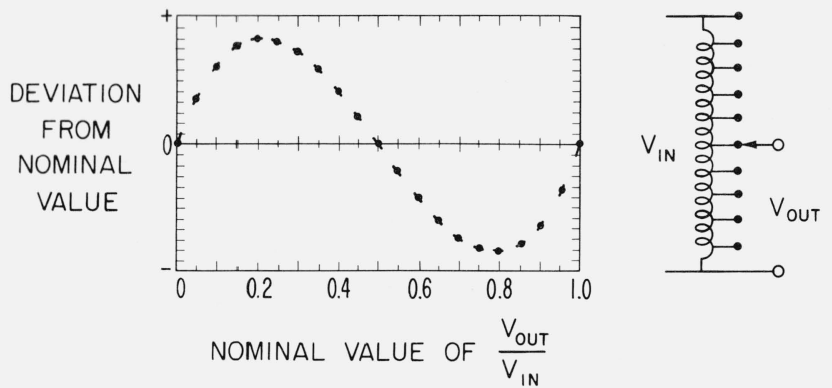

Figure 3. Typical error curve for one decade of a low-frequency decade voltage divider.

Each section of this divider accurately divides its input voltage in half to give an attenuation of $6 \mathrm{~dB}{ }^{1}$ An attenuator made with $n$ sections will have approximately $6 n \mathrm{~dB}$.

Figure 2c shows an adjustable ratio "binary" inductive voltage divider. The principle of operation is the same as for low frequency decade voltage dividers except that each section divides its input voltage into only two equal parts instead of 10 equal parts. T. L. Zapf et al., have shown that the error curve for any one isolated decade of a low frequency decade voltage divider has an "S" shape such as shown in figure $3[1] .{ }^{2}$ As the frequency increases, the magnitude of the error increases but the curve still tends to go through zero at a ratio of 0.5 . It is this error curve that suggested that $2: 1$ ratio dividers would be accurate at high frequencies as well as low frequencies. If $n$ of these $2: 1$ ratio dividers are connected as shown in figure $2 \mathrm{c}$ the voltage ratio is

$$
\frac{V_{\text {out }}}{V_{\text {in }}}=\sum_{i=1}^{n} \frac{\delta_{i}}{2^{i}}, \delta_{i}=\left\{\begin{array}{l}
1 \text { if the } i \text { th switch is up } \\
0 \text { if the } i \text { th switch is down. }
\end{array}\right.
$$

The input of each section is connected either across the top half or across the bottom half of the preceeding section. To obtain the same resolution with a binary divider as with a decade divider requires that the binary divider have approximately 3 times as many sections as the decade divider has.

Figure $2 \mathrm{~d}$ shows three basic $2: 1$ ratio dividers connected in series with two more $2: 1$ ratio dividers connected so as to assure that the same voltage is applied to each of the three in series. Any number could be connected in series, however the combination shown gives the very useful ratios

$$
\frac{V_{1}}{V_{2}}=1,2, \text { and } 5 \text {. }
$$

This type of divider could be used in a bridge such as shown in figure $2 \mathrm{a}$ to compare standards of impedance which are often built to have nominal values in a 1-2-5 sequence.

\footnotetext{
More accurately $20 \log 2=6.020600 \mathrm{~dB}$

${ }^{2}$ Figures in brackets indicate the literature references at the end of this paper.
}

\section{The 2 : 1 Ratio Divider}

The $2: 1$ ratio divider is a center-tapped transformer that divides a voltage into two equal parts. It is convenient to define the complex ratio error, $\rho=\rho_{r}+j \rho_{i}$, as the error in the ratio of the output voltage to the input voltage. The output voltages $e_{1}$ and $e_{2}$ shown in figure 1 then become

$$
e_{1}=\frac{e_{i}}{2}(1+\rho), \quad e_{2}=\frac{e_{i}}{2}(1-\rho) .
$$

If $|\rho| \ll 1$, the ratio $e_{1} / e_{2}$ is approximately

$$
\frac{e_{1}}{e_{2}}=1+2 \rho .
$$

To get an expression for the ratio error, consider the divider as two impedances in series which differ by a small amount $\Delta Z$ as shown in figure 4 . If no current is drawn from the center tap, the voltage ratio $e_{1} / e_{2}$ is

$$
\frac{e_{1}}{e_{2}}=\frac{Z+\Delta Z}{Z}=1+\frac{\Delta Z}{Z}
$$

From (1) and (2) we get

$$
\rho=\frac{\Delta Z}{2 Z}=\frac{\Delta R+j \omega \Delta L}{R+j \omega L}
$$

where $R+j \omega L$ is the open circuit input impedance, $Z_{\text {in }}$, of the transformer. The real and imaginary components of $\rho$ obtained from (3) are

$$
\begin{aligned}
& \rho_{r}=\frac{R \Delta R+\omega^{2} L \Delta L}{R^{2}+\omega^{2} L^{2}} \\
& \rho_{i}=\frac{\omega R \Delta L-\omega L \Delta R}{R^{2}+\omega^{2} L^{2}} .
\end{aligned}
$$

If $Q^{2} \gg 1$ as is often the case, where $Q \equiv \omega L / R$, (4) and (5) reduce to

$$
\begin{aligned}
& \rho_{r}=\frac{\Delta L}{L}+\frac{\Delta R}{\omega L Q} \\
& \rho_{i}=-\frac{\Delta R}{\omega L}+\frac{\Delta L}{L Q} .
\end{aligned}
$$

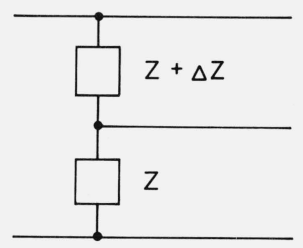

FIGURE 4. Simplified circuit of a 2:1 ratio divider. 
These equations show the importance of winding the transformer so that it has a large inductance. The value of $\Delta L / L$ is made small by winding the transformer on a high permeability core to increase $L$, and by twisting the wires to minimize $\Delta L$. A twisted cable is made by folding one wire in half and twisting the pair fairly tightly with a hand drill as shown in figure $5 \mathrm{a}$. This cable is wound on the magnetic core and connected as shown in figure $5 \mathrm{~b}$. The wires are connected so that the current path from the upper tap to the center tap is the same as the current path from the center tap to the bottom tap. Thus, both windings link very nearly the same magnetic flux and have a small difference in inductance $\Delta L$. Twisting the wires helps assure that both windings link the same flux.

Figure 6 shows the variation of $\rho$ with frequency for a transformer having 42 turns of twisted No. 26 magnet wire wound on a ferrite torodial core. Note that $\rho_{r}$ is essentially constant with frequency up to $2 \mathrm{MHz}$ which is the upper frequency limit of our bridge. To see how high in frequency $\rho_{r}$ will remain constant, we need to look at the equivalent circuit of the divider.

Figure 7 shows one of two exact equivalent circuits for an autotransformer [2] which is what the 2:1 ratio divider is. The $Y_{\text {oc }}$ is the open-circuit admittance measured across the input terminals and $Z_{\mathrm{sc}}$ is the short-circuit "leakage" impedance measured across either output with the input terminals short-circuited. It can be seen from figure 7 that the voltage ratio $V_{1} / V_{2}$ at the output terminals will be equal to $e_{1} / e_{2}$ only if no current flows through $Z_{\mathrm{sc}}$. If within the transformer housing the admittance from A to B differs from that from $\mathrm{B}$ to $\mathrm{D}$ by an amount $\Delta Y_{t}$, and if $\left|Z_{\mathrm{sc}} \Delta Y_{t}\right| \ll 1$, then

$$
\frac{V_{1}}{V_{2}}=\frac{e_{1}}{e_{2}}-2 Z_{\mathrm{sc}} \Delta Y_{t}
$$

If $\Delta Y_{t}$ is mainly a difference in capacitance, $\Delta C_{t}$,
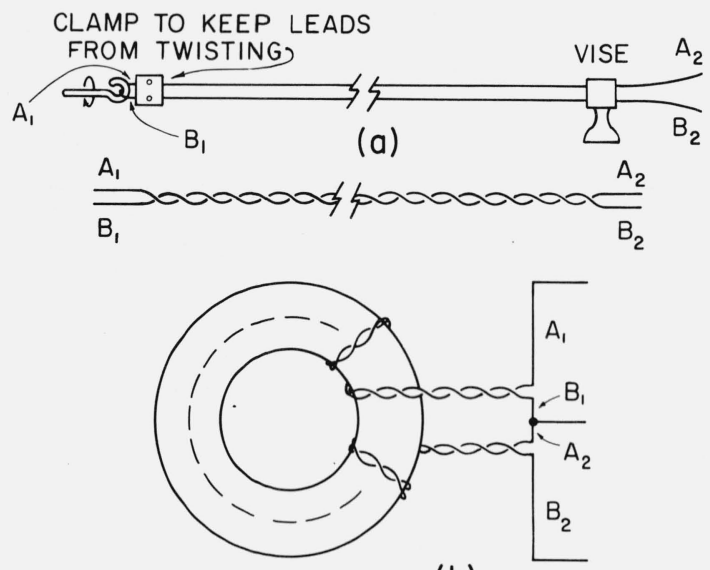

(b)

FigURE 5. The wires are twisted, wound on the core and connected as shown here to make a 2:1 ratio divider.

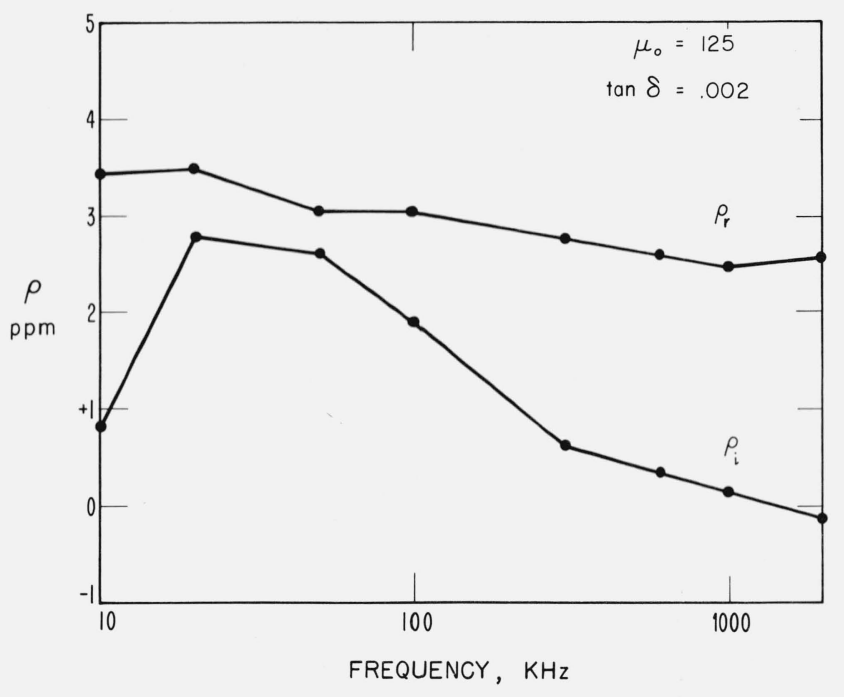

FIGURE 6. Error curve for one of the first experimental dividers showing how $\rho_{\mathrm{r}}$ and $\rho_{\mathrm{i}}$ are relatively constant with frequency.

$$
\frac{V_{1}}{V_{2}}=1+2 \rho+2 \omega^{2} L_{\mathrm{sc}} \Delta C_{t}-j 2 R_{\mathrm{sc}}^{\cdot} \omega \Delta C_{t}
$$

where $L_{\mathrm{sc}}$ is the leakage inductance defined by $Z_{\mathrm{sc}}$ $\equiv R_{\mathrm{sc}}+j \omega L_{\mathrm{sc}}$. A typical value of $L_{\mathrm{sc}}$, such as for the transformer whose ratio error is shown in figure 6 , is $L_{\mathrm{sc}}=0.1 \mu \mathrm{H}$. By using trimmer capacitors from A to $\mathrm{B}$ and $\mathrm{B}$ to $\mathrm{D}, \Delta C_{t}$ can be made less than $0.01 \mathrm{pF}$ giving, in ppm,

$$
2 \omega^{2} L_{\mathrm{sc}} \Delta C_{t}<0.1 f_{\mathrm{MHz}}^{2}
$$

and

$$
2 R_{\mathrm{sc}} \omega \Delta C_{t}<0.01 f_{\mathrm{MHz}}
$$

where $f_{\mathrm{MHz}}$ is the frequency in $\mathrm{MHz}$. Since this loading error is proportional to the frequency squared, it will be the predominant error term at frequencies much above $1 \mathrm{MHz}$. The transformer whose characteristic is shown in figure 6 has trimmer capacitors across the output leads to adjust $\Delta C_{t}$ to a minimum. Without adjusting $\Delta C_{t}$ to a minimum the $\rho_{r}$ curve increases to a value of about $5 \mathrm{ppm}$ at $2 \mathrm{MHz}$.

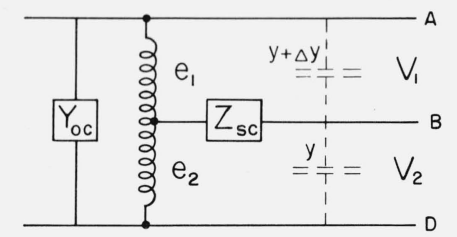

Figure 7. An "exact" equivalent circuit for an autotransformer with lead admittances $\mathrm{Y}$ and $\mathrm{Y}+\Delta \mathrm{Y}$ added. 


\section{A Bridge to Measure $\rho$}

A simplified diagram of the bridge used to measure $\rho$ is shown in figure 8. The two arms of the transformer are balanced against two capacitors $C^{\prime}$, each having a nominal value of $1000 \mathrm{pF}$. The bridge is balanced with the capacitance $C$ and the conductance $G$. The transformer is then removed from the bridge, rotated so that D is at the top and A is at the bottom, and then replaced on the bridge. The bridge is again balanced as before, changing $C$ an amount $\Delta C$ and $G$ an amount $\Delta G$. Solving the bridge equations gives

$$
\begin{gathered}
\rho_{r}=\frac{\Delta C}{4 C^{\prime}} \\
\rho_{i}=\frac{-\Delta G}{4 \omega C^{\prime}}
\end{gathered}
$$

where it has been assumed that $|\rho| \ll 1, C^{\prime \prime} \ll C^{\prime}$, and $G^{\prime \prime 2} \ll \omega^{2} C^{\prime 2}$.

A more detailed diagram of the bridge is shown in figure 9a. The capacitors $C$ and $C^{\prime}$ are three-terminal capacitors having admittances to ground shown as dotted lines. If these admittances are unequal they will load the transformer causing currents to flow through $Z_{\text {sc }}$ and, thereby, change the apparent voltage

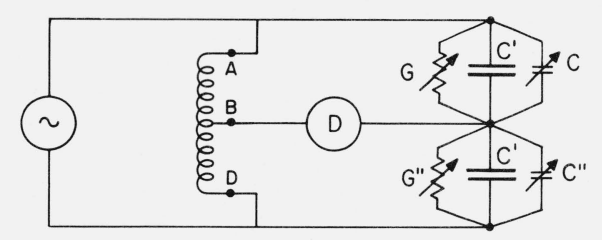

FIGURE 8. Simplified circuit of a bridge to measure the ratio error of a 2:1 ratio divider.

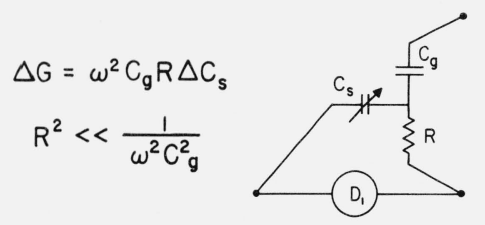

(b)

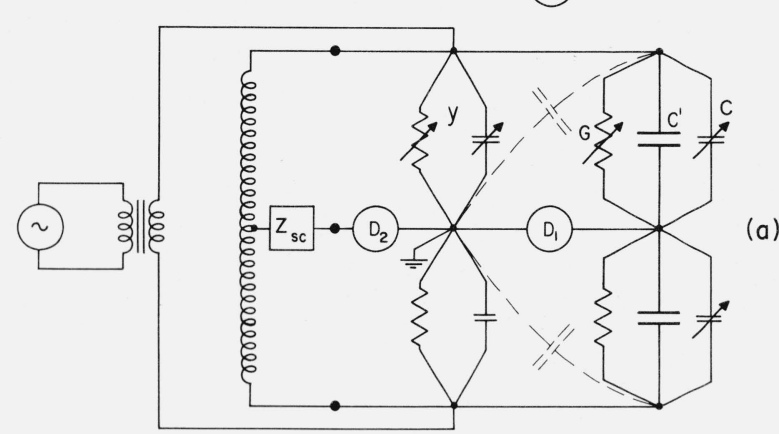

Figure 9. A more detailed drawing of the circuit shown in figure 8. ratio of the transformer. To measure the true voltage ratio $e_{1} / e_{2}$ of the transformer, a second detector, $D_{2}$, is placed between the center tap of the transformer and ground in order to balance the admittances to ground with $Y$. With both detectors nulled for both positions of the transformer, the bridge equations yield (10) and (11) as before.

To measure the small changes in conductance, $\Delta G$, the T-network shown in figure $9 \mathrm{~b}$ was used for $G$. Changing $C_{s}$ an amount $\Delta C_{s}$ gives a change in conductance

$$
\Delta G=\omega^{2} C_{y} R \Delta C_{s}
$$

and a change in capacitance which is negligible compared to $\Delta C$. Putting (12) into (11) gives

$$
\rho_{i}=-\frac{R C_{g}}{4 C^{\prime}} \omega \Delta C_{s}
$$

The resolution for $\rho_{r}$ and $\rho_{i}$ is $0.025 \mathrm{ppm}$ from $1 \mathrm{kHz}$ to $1 \mathrm{MHz}$ and $0.25 \mathrm{ppm}$ to about $3 \mathrm{MHz}$. A photograph of the bridge is shown in figure 10. Bridges of similar design are described in references [3] and [4].

\section{Optimum Transformer Design}

Using the bridge shown in figure 10, a number of experiments were made to determine the optimum coil geometry, core material, type of wire, wire sizes, and amount of twist of the wire.

\subsection{Coil Geometry}

Theoretically the best coil geometry would be that which gives a maximum inductance for a minimum

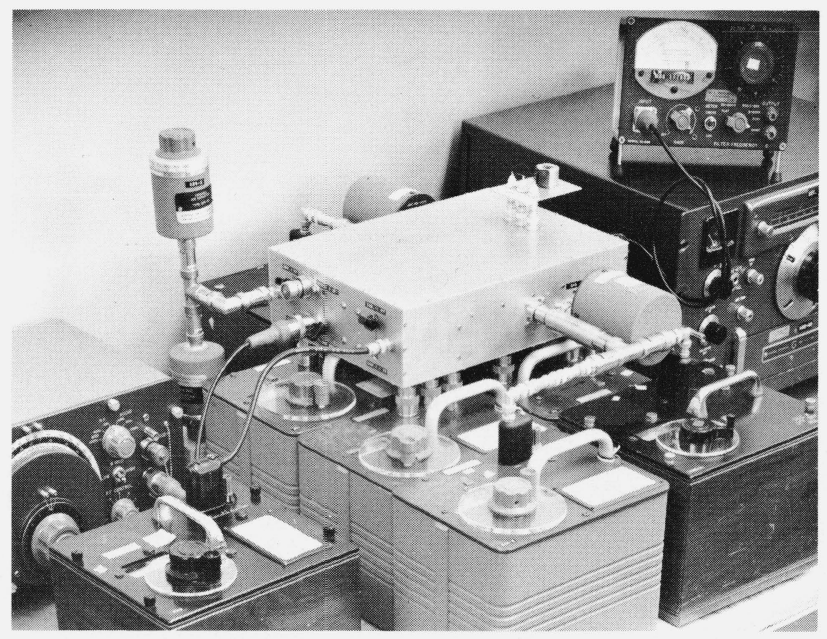

FIGURE 10. Photograph of bridge components, showing a divider, with its shield removed, connected at the top of the bridge. 
length of cable. Large inductance is needed to decrease $\rho_{r}$, and minimum cable length is needed to make $L_{\mathrm{sc}}$ small. The leakage inductance per unit length of cable is given approximately by

$$
\frac{L_{\mathrm{sc}}}{l}=0.2 \frac{P}{d} \tan ^{-1} \frac{d}{P} \mu \mathrm{H} / \mathrm{m}
$$

where

$$
\begin{aligned}
P & =2 t+\delta \\
t & =\text { wire insulation thickness } \\
d & =\text { wire diameter } \\
\delta & =\text { skin depth }\left(\leqslant \frac{d}{2}\right) \\
l & =\text { cable length. }
\end{aligned}
$$

If $\delta>d / 2$, use $d / 2$ in place of $\delta$. Equation (14) is actually the inductance per unit length of two long parallel thin tapes of width $d$ spaced a distance $P$ apart. By defining $P$ and $d$ as stated under (14), the results from (14) agree with measured data to within 10 percent which is sufficiently accurate for most applications. Note that $L_{\mathrm{sc}}$ is independent of the core permeability. To decrease $L_{\mathrm{sc}}$ it is necessary to decrease the cable length. Changing the wire diameter or insulation thickness has only a secondary affect on $L_{\mathrm{sc}}$. Measured values of $L_{\mathrm{sc}}$ and $R_{\mathrm{sc}}$ from $100 \mathrm{kHz}$ to $2 \mathrm{MHz}$ are shown in figure 11 for No. 24 wire. The leakage inductance decreases slightly due to skin effect. The resistance $R_{\mathrm{sc}}$ increases slightly faster than the square root of frequency primarily due to skin effect.

\subsection{Core Material}

Figure 12 shows how $\rho_{r}$ is decreased by increasing the permeability $\mu$. These four transformers were identical except for the type of core material used. One long twisted cable was made and cut in half. One half was used to wind No. 10 and the other half to wind No. 11. Transformers Nos. 12 and 13 were, likewise, made from one cable cut in half. The results show that $\rho_{r}$ is inversely proportional to $\mu$. The cores were all very low-loss ferrite toroids with loss tangents of about 0.002 . Cores which are very lossy do not give the same results. The $Q$ for transformers with lossy cores is so low that the second term on the right of (6) may become larger than the first. The second term is a function of the core loss as well as the permeability. The core material, therefore, should have a high $\mu$ but not be too lossy. The core material can be fairly lossy if $\Delta R$ is small enough to make the second term on the right of (6) negligible.

\subsection{Type of Wire}

A number of experiments with different types of wire led to the conclusion that wire which has a very

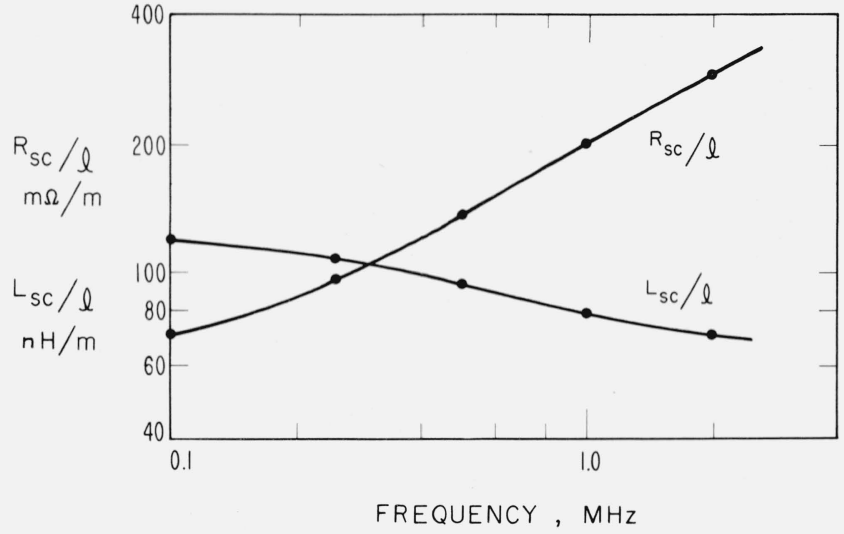

FiguRE 11. Variation of the leakage inductance and resistance with frequency.

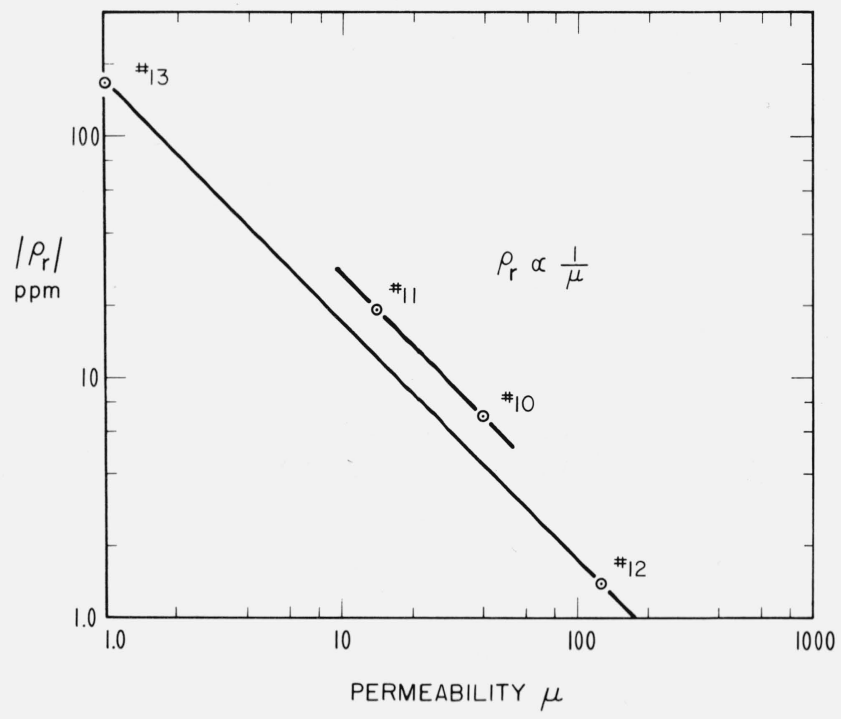

FIGURE 12. Ratio error versus core permeability for four transformers made identical except for core permeability.

uniform resistivity and diameter gives the lowest $\Delta R$ values. Figure 13 shows the errors for four transformers which are identical except for the type of wire used. These transformers have air cores to eliminate the effect of magnetic core properties. Another reason for using air core transformers is that the errors are larger and easier to measure. The shape of the single layer solenoid type winding is shown in figure 14. The different types of wire used were formvar coated magnet wire $(C)$, TEFLON ${ }^{\circledR}$ coated magnet wire $(\mathrm{Al} \& \mathrm{~A} 3)$, and copper thermocouple wire (T). Note that the thermocouple wire $\rho_{r}$ curve is constant with frequency and quite small for an air core. This means that the second term in (6) is negligible even though the $Q$ is fairly low. Using thermocouple wire we were consistently able to wind transformers with low errors which are constant with frequency. 
The thermocouple wire transformers are true "inductive" voltage dividers. The other transformers are more nearly "resistive" dividers than "inductive" dividers. The inductance of each of these four transformers is about $15 \mu \mathrm{H}$. Since $\rho_{r} \doteq 10 \mathrm{ppm}$ for the thermocouple wire transformer, (6) gives $\Delta L$ $\doteq 150 \mathrm{pH}$. Typical values of $\Delta L$ are 100 to $500 \mathrm{pH}$. They are constant with frequency and independent of $\mu$. Expressing $L$ as $L=\mu N^{2} L_{a}$, (6) becomes

$$
\rho_{r} \doteq \frac{\Delta L}{\mu N^{2} L_{a}}
$$

where $N=$ number of turns on the core and $L_{a}$ is the geometric inductance of the core. Typical values of $\Delta R$ are 50 to $500 \mu \Omega$ for thermocouple wire and 10 times that for the other wire we tried. These figures are all for No. 24 wire.

It should be noted that any wire, including thermocouple wire, generally gives larger errors if the wire is stretched. A number of transformers were made with wire that was stretched approximately 1 percent to remove slight kinks in the wire. The errors were significantly larger than for transformers made from the same spool of wire with the slight kinks left in the wire. It is best to purchase wire wound on large spools so that it is relatively free of kinks when removed from the spool.

\subsection{Wire Size}

The experiment described above was repeated with No. 20 wire and also with No. 30 wire. The larger

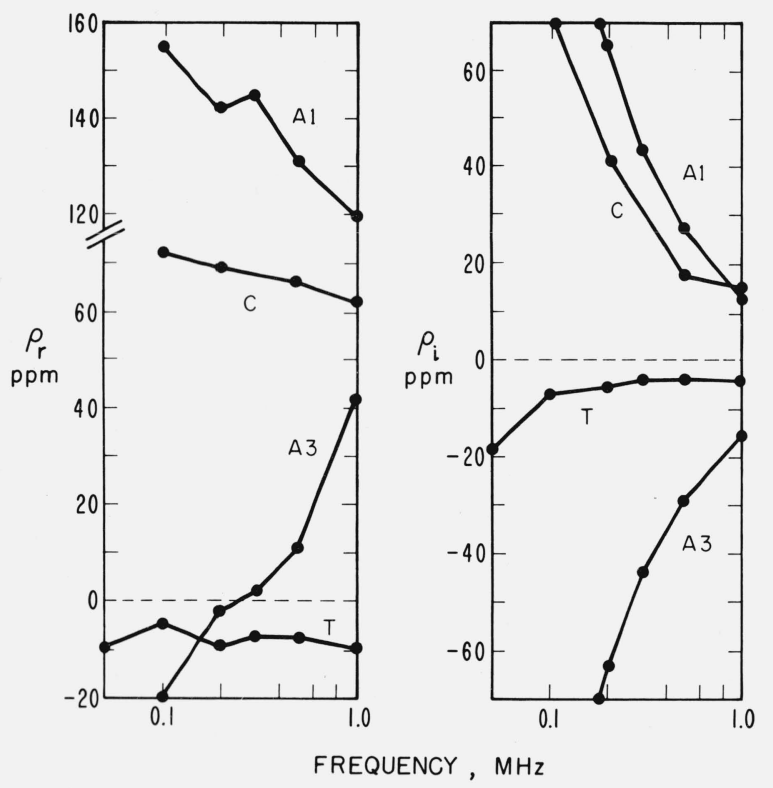

FigURE 13. Comparing results from four different types of No. 24 wire, where $A 1$ and $A 3$ are TEFLON ${ }^{\circledR}$ coated magnet wire with single $(0.0006 \mathrm{in})$ and triple $(0.0015$ in $)$ insulation thickness; $C$ is formvar coated magnet wire, and $T$ is enamel coated copper thermocouple wire. wire transformers gave slightly lower $\rho_{i}$ errors than the corresponding No. 24 wire transformers gave. There was no significant decrease in the $\rho_{r}$ errors. The results are shown in figure 15. Transformers made with No. 30 wire had considerably higher errors than those made with No. 24 or No. 20 wire.

\subsection{Cable Twist}

To determine how much the wires should be twisted, a number of transformers were made identical except for the amount the wires were twisted. Figure 16a shows $\rho_{r}$ and $\rho_{i}$ plotted against the number of turns per unit length of the cable. The length, $l_{T}$ of one turn is defined in figure 16b. These are all air core transformers wound with No. 24 thermocouple wire. The dots are single layer solenoid windings whereas the $X$ 's are multilayer coils wound on nylon bobbins. The inductance, $L$, of each transformer is approximately $15 \mu \mathrm{H}$. As can be seen from figure 16, any twist from four to ten turns/inch (160 to 400 turns/meter) gives about the same results. Below four turns/inch the error increases. At about 12 turns/inch (500 turns/ meter) the wire breaks. In terms of the twist length, $l_{T}$, and wire diameter, $d$, satisfactory results were obtained in the range

$$
0.08<\frac{d}{l_{T}}<0.2 \text {. }
$$



Figure 14. An air core, solenoid type winding. 


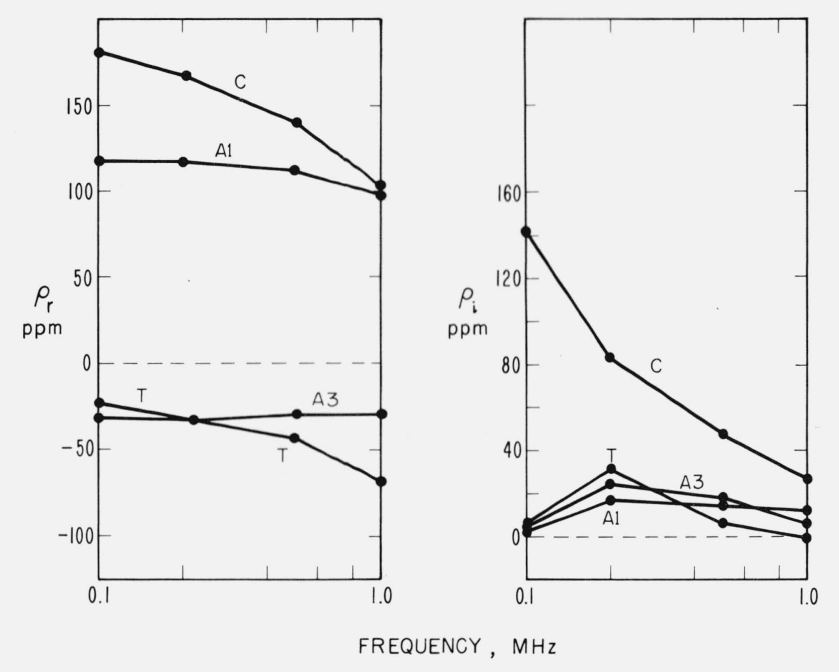

FigURE 15. Comparing results from four different types of No. 20 wire where $A 1, A 3, C$, and $T$ are as defined in figure 13.

This equation should also be a reasonable guide for twisting wires other than size No. 24.

\subsection{Lead Connections}

One more important consideration in winding the transformers is how the leads are connected. The first transformers that we wound were connected as shown in figure $17 \mathrm{a}$. The ratio errors were quite unstable and very sensitive to slight movement of the leads. To find a better way of connecting the leads, a number of air core transformers were made identical except for the lead connections. Figure 17 shows the three different ways the leads were connected. Of these three ways, the one shown in figure $17 \mathrm{c}$ was most stable and gave the smallest errors. The improvement in stability is probably due to the smaller mutual inductance and capacitance between leads. The lower error is probably a result of the ease with which the corresponding leads can be cut to the same length. It is important that in any arrangement of the leads that the leads on one side of the center tap be the same length as the corresponding leads on the other side. A difference in lead length of 0.001 in will give a $\Delta L$ of about $40 \mathrm{pH}$ for No. 24 wire.

\subsection{Cup-Core Design}

One of the easiest ways of constructing a transformer with magnetic cores is to wind the cable on a bobbin and then clamp magnetic cup cores to the bobbin such as shown in figure 18 . Figures 19 and 20 show the errors for three cup core transformers constructed like the one shown in figure 18 . These transformers were made with all the optimum parameters that were found. The cables were made with No. 24 thermocouple wire twisted to six turns/inch (240 turns/meter). The cores

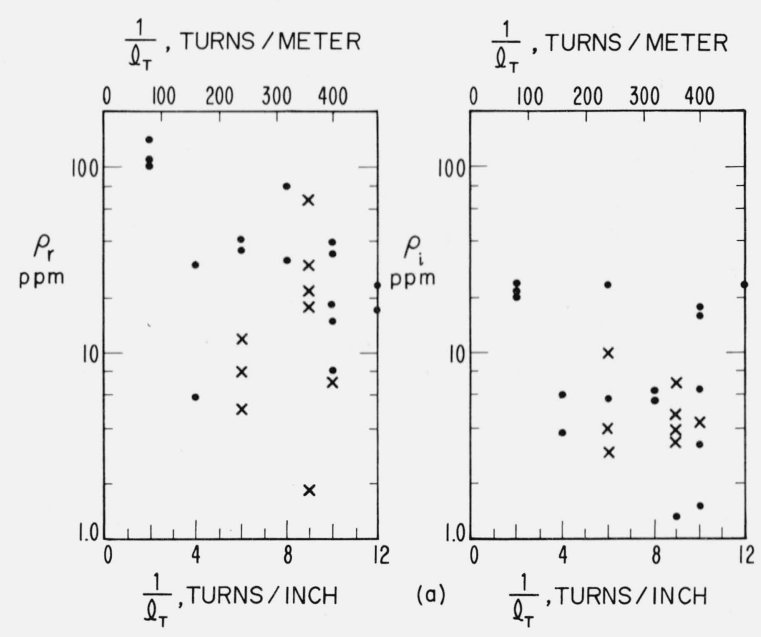

(b)



FigURE 16. Results of air core solenoid type (dots) and bobbin type $(X$ 's ) transformers wound with cables twisted a different number of turns per inch.

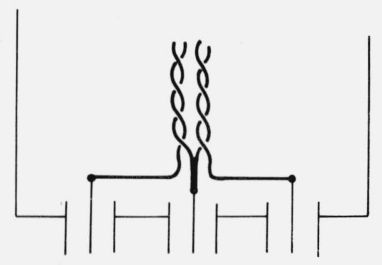

(a)

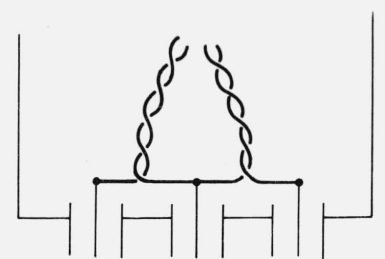

(b)

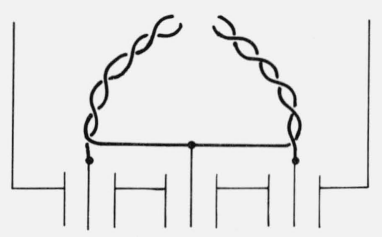

(c)

FigURE 17. Three different arrangements of the transformer leads.

are high permeability, fairly low-loss ferrite cores. The increase in $\rho_{r}$ and $\rho_{i}$ at frequencies above $100 \mathrm{kHz}$ is due to the capacitance between leads not being equal. If these capacitances were balanced as discussed in part 3 , the errors would be less than $1 \mathrm{ppm}$ to above $1 \mathrm{MHz}$.

With care, the error can be made less than $0.1 \mathrm{ppm}$. A fourth cup core transformer was adjusted to have a $\rho_{r}$ error less than $0.1 \mathrm{ppm}$ at $100 \mathrm{kHz}$ by changing the length of one lead slightly. Then $\rho_{r}$ was adjusted to less than $0.1 \mathrm{ppm}$ at $1 \mathrm{MHz}$ by adjusting small trimmer capacitors placed between the leads. As figure 21 shows, the resulting $\rho_{r}$ error is less than $0.1 \mathrm{ppm}$ from $30 \mathrm{kHz}$ to above $1 \mathrm{MHz}$. 


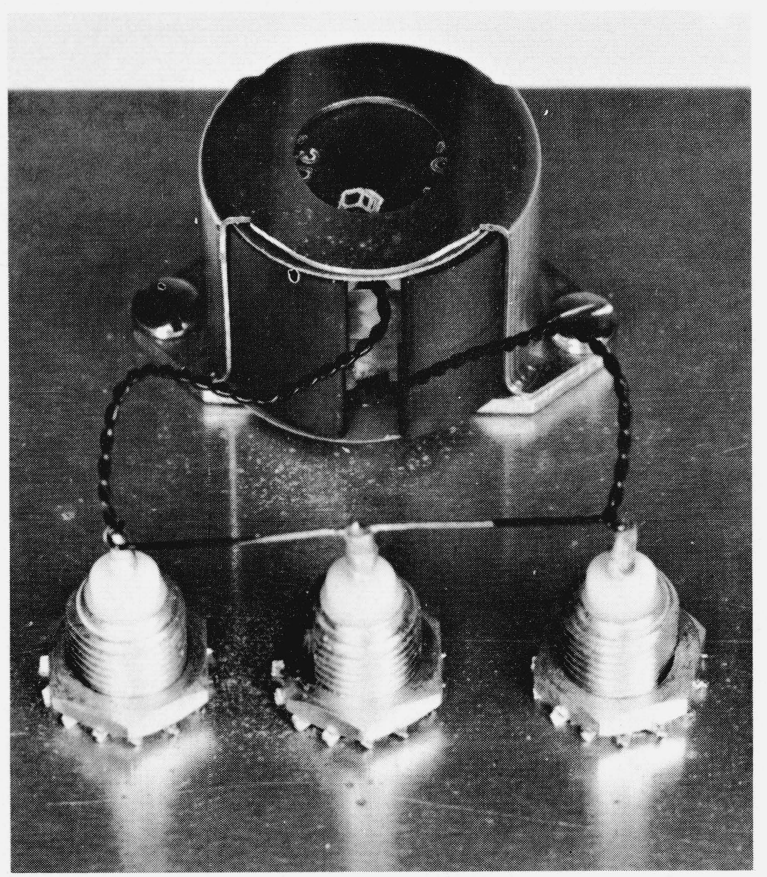

FIGURE 18. A cup-core transformer having very low ratio errors like that shown in figures 19 and 20.

The cover of the shield is not shown.

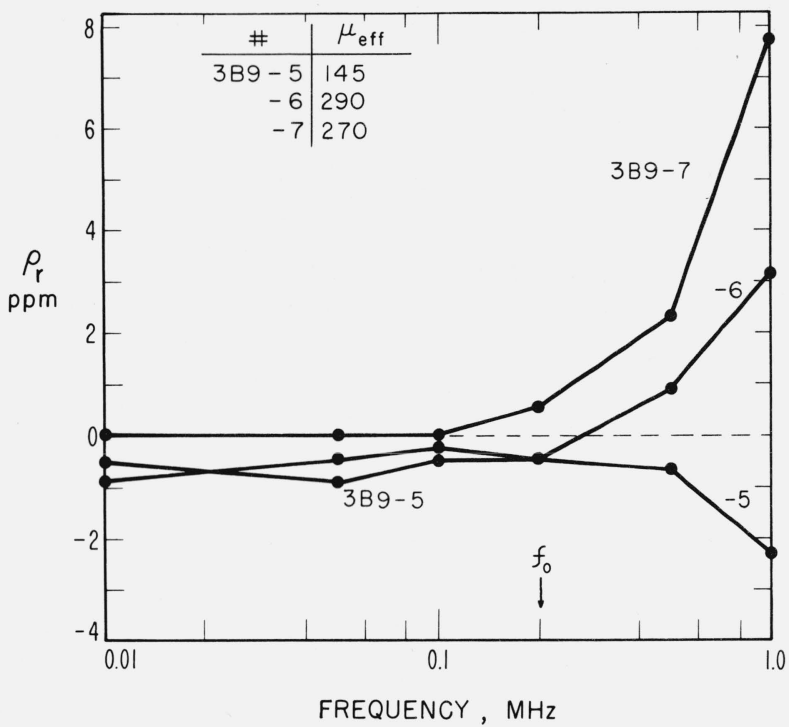

FIGURE 19. Results from three cup-core transformers wound with No. 24 copper thermocouple wire twisted to six turns/inch.

\subsection{Tape-Core Transformers}

Very broad band 2:1 ratio dividers can be made with high permeability magnetic tape cores. Figure 22 shows the ratio error of a divider wound on a supermalloy tape core having a tape thickness of $1 \mathrm{mil}$. The

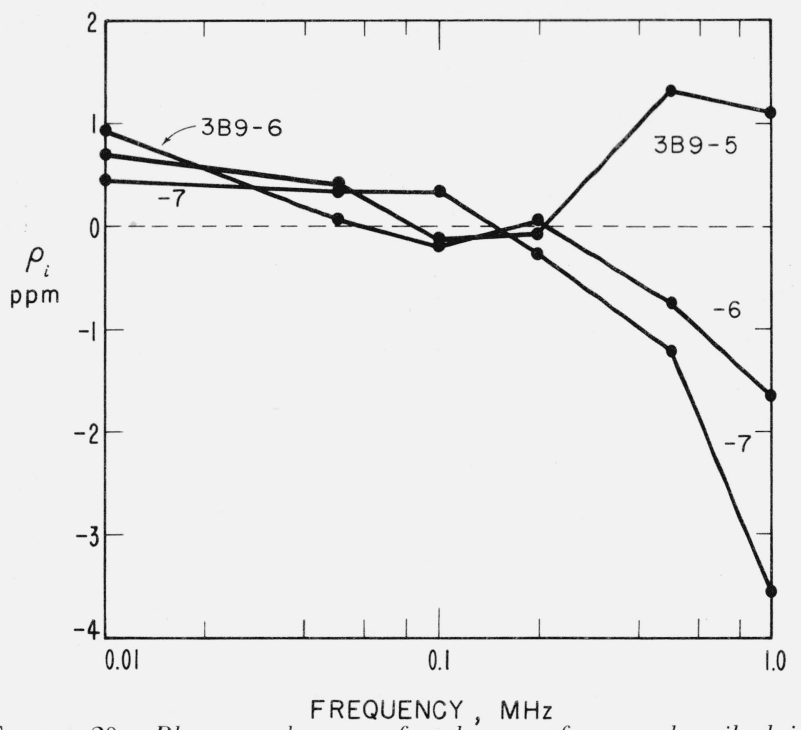

FIGURE 20. Phase angle error for the transformers described in figure 19.

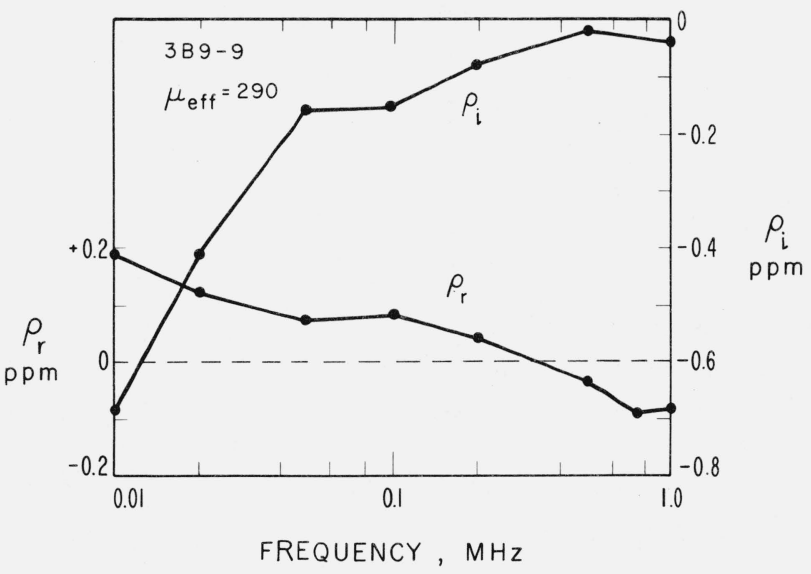

FIGURE 21. Ratio error of a cup core transformer which has been adjusted to have a minimum $\rho_{\mathrm{r}}$ by adjusting lead length and by balancing the capacitance between leads.

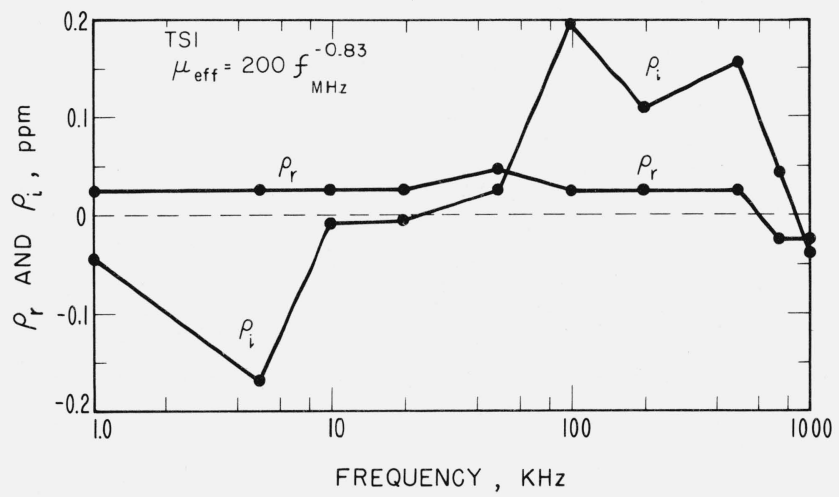

FigURE 22. Ratio error of a transformer wound on a supermalloy. 1 mil tape core having dimensions $O D=1.150, I D=0.650$ and $H=0.375$ in . 
core has 64 turns of No. 24 copper thermocouple wire twisted to about 8 turns/inch (320 turns/meter). The capacitance between leads has been adjusted to be equal. The $\rho_{r}$ error is less than 5 parts in $10^{8}$ from $1 \mathrm{kHz}$ to $1 \mathrm{MHz}$.

\section{Conclusion}

The 2:1 ratio transformer is a simple, yet accurate, divider which is easy to construct. Using the optimum parameters discussed in part 5 , it is easy to keep the ratio error less than $1 \mathrm{ppm}$ from $1 \mathrm{kHz}$ to $1 \mathrm{MHz}$. Air core transformers having no magnetic cores can be made with errors less than $10 \mathrm{ppm}$ over a reduced frequency range. With extra care, $\rho_{r}$ can be made less than $0.1 \mathrm{ppm}$ to above $1 \mathrm{MHz}$. The ratio errors are quite insensitive to temperature, time, shock, and most any change in the environment since both windings of the transformer are influenced equally by any change in the environment.
Five cup-core transformers such as described in part 5.7 have been combined as shown in figure $2 b$ to make an accurate $30 \mathrm{~dB}$ attenuator at $1 \mathrm{MHz}$. Details of this type of attenuator together with a complete error analysis will be given in a future publication.

\section{References}

[1] Zapf, T. L., C. H. Chinburg, and N. K. Wolf (September 1963), Inductive voltage dividers with calculable relative corrections, IEEE Trans. on Instrumentation and Measurement IM-12, 80-85.

[2] EE Staff, MIT (1943), Magnetic circuits and transformers (John Wiley \& Sons, Inc., New York, N.Y.), 400.

[3] McGregor, M. C., J. F. Hersh, R. D. Cutkosky, F. K. Harris, and F. R. Kotter (Dec. 1958). New apparatus at the National Bureau of Standards for absolute capacitance measurement, IRE Trans. on Instrumentation I-7, No. 3 and 4, 253-26l.

[4] Cutkosky, R. D., and J. Q. Shields (September 1960), The precision measurement of transformer ratios, IRE Trans. on Instrumentation I-9, No. 2, 243-250.

(Paper 71C2-249) 